\title{
GPR applications for geotechnical stability of transportation infrastructures
}

\author{
A. Benedetto ${ }^{\mathrm{a} *}, \mathrm{~F}$. Benedetto ${ }^{\mathrm{b}}$ and F. Tosti ${ }^{\mathrm{a}}$ \\ ${ }^{a}$ Department of Sciences of Civil Engineering, University of Roma Tre, Via Vito Volterra 60, \\ Rome, 00146, Italy; ${ }^{b}$ Department of Applied Electronics, University of Roma Tre, \\ Via della Vasca Navale, Rome 00146, Italy
}

\begin{abstract}
Nowadays, severe meteorological events are always more frequent all over the world. This causes a strong impact on the environment such as numerous landslides, especially in rural areas. Rural roads are exposed to an increased risk for geotechnical instability. In the meantime, financial resources for maintenance are certainly decreased due to the international crisis and other different domestic factors. In this context, the best allocation of funds becomes a priority: efficiency and effectiveness of plans and actions are crucially requested. For this purpose, the correct localisation of geotechnically instable domains is strategic. In this paper, the use of Ground-Penetrating Radar (GPR) for geotechnical inspection of pavement and sub-pavement layers is proposed. A three-step protocol has been calibrated and validated to allocate efficiently and effectively the maintenance funds. In the first step, the instability is localised through an inspection at traffic speed using a $1-\mathrm{GHz}$ GPR horn launched antenna. The productivity is generally about or over $300 \mathrm{Km} /$ day. Data are processed offline by automatic procedures. In the second step, a GPR inspection restricted to the critical road sections is carried out using two coupled antennas. One antenna is used for top pavement inspection (1.6 GHz central frequency) and a second antenna ( $600 \mathrm{MHz}$ central frequency) is used for sub-pavement structure diagnosis. Finally, GPR data are post-processed in the time and frequency domains to identify accurately the geometry of the instability. The case study shows the potentiality of this protocol applied to the rural roads exposed to a landslide.
\end{abstract}

Keywords: landslides; ground-penetrating radar (GPR); pavement and sub-pavement layers; instability

\section{Introduction}

Starting from the nineties, the consciousness that climate is somehow changing is growing in the scientific community and among the general public. Severe meteorological events, such as storms, intense rainfall, tornados as well as long periods of drought, are increasingly frequent all over the world.

One of the most critical consequences is the strong and rapid variations of water content in soils. The increase of ground water pressure reduces the friction among soil particles, thus increasing the geotechnical instability and the landslide risk.

Rural road networks are mainly exposed to landslide risk. Moreover, in recent decades, the maintenance of rural environments is reduced. This has made the rural infrastructure systems much more vulnerable to very intense meteorological events. 
In addition, the financial resources for maintenance are certainly decreased due to the international crisis and other different domestic factors. In this framework, reliable instruments, efficient methods and effective solutions for prevention and rehabilitation are requested.

Falls, topples, slides, flows and spreads are basically the types of landslide as identified by Varnes in 1978 [1]. Among them, flows are very frequently responsible for severe road damages. Following the geotechnical definition, flow is a spatially continuous movement in which surfaces of shear are short-lived, closely spaced and usually not preserved. The distribution of velocities in the displacing mass resembles that in a viscous liquid. The lower boundary of a displaced mass may be a surface along which an appreciable differential movement or a thick zone of distributed shear has taken place [2].

In this paper, we refer to the flows on the basis of the assumption that flows occur as the water content in the sliding mass of soil is much greater than the water content of the bedrock on which the soil slides. Following this assumption, the landslide can be detected and the geometry of the volume of the soil mass can be delimited and monitored by measuring the water content variation in the soil.

Ground-penetrating radar (GPR) has been used for geological and geotechnical engineering applications for the last few years. For an accurate and expeditious monitoring of landslides areas, GPR has been used in the Carpathian Mountains in the eastern part of the Czech Republic, where the landslide risks are high. The outcomes of the work were satisfactory, in terms of effectiveness and efficiency [3]. GPR has also been used to monitor ancient landslides, from past seismic activities, in Alaska [4]. To improve information on landslides, GPR data are integrated with information from other equipments, such as seismic refraction and electrical resistivity [5]. The case of falling rocks is discussed in the research project 'Landslide Hazard Assessment and Cultural Heritage' [6]. In this case, prediction models have been developed. Ékes and Friele [7] presented two case studies where GPR was used as the chief investigative tool to understand the underlying cause for road failures as they relate to fill slope stability in the Coast Mountains of British Columbia, Canada. The authors demonstrated that GPR is an efficient, nondestructive and cost-effective tool for characterising the nature of slope stability problems. In general, GPR in all of these cited cases has been used to detect rock fractures and soil discontinuities. These applications are based on the analysis of multiple GPR signal reflections from discontinuous surfaces in the time domain.

GPR is used to identify the interlayers sliding surface and to monitor as the soil moisture varies in time and space, following the assumption that a high water content variation between a sliding soil mass and a bedrock is expected, in the case of flows detection. A few techniques have been used in the past two decades to evaluate the water content in soils and specifically in sub-asphalt soils. Traditionally, the sub-asphalt water content is often estimated via gravimetric sampling, time-domain reflectometry, neutron probe logging and measuring the capacitance or resistance of devices. These methods are accurate, but they also have many disadvantages. Considering these limitations, many authors proposed to measure the moisture content using GPR. With GPR it is possible to collect data quickly along the road and it is reasonably possible to obtain the value of the volumetric water content of soil in unsaturated porous media, such as sub-asphalt soil [8].

Basically the moisture content is evaluated using the relationship between the dielectric constant of a soil and its volumetric water content. Various empirical correlations have been proposed, such as the well-known equation suggested by Topp [9], which is supposedly valid for any type of soil. Reviews of the various theoretical models are available [10-16]. Another theoretical approach that relates the soil water content and 
the permittivity is based on dielectric mixing [17]. Other models are based on estimations of the dielectric permittivity, by computing the time delay of GPR signal reflections once the value of the wave propagation velocity in the medium is determined $[18,19]$. In any case, these methods require calibration steps.

The moisture content is also estimated considering the reduction of the amplitude of reflections from the sub-asphalt layers in relation to the amplitude of reflection from a large metal plate [20]. Wet soil absorbs signal energy, thus reducing the amplitude of the reflections.

In addition, a self-consistent approach based on signal processing in the frequency domain is also used here. This approach is focusing on the Rayleigh scattering [21].

In this paper, we present a novel method for landslide inspection to reduce the risk of damage of rural roads.

\section{Objectives}

The main objective of this research is to reduce the risk of damage of rural roads, which is induced by landslides, and to improve the operations of maintenance and rehabilitation through effective and efficient inspections and preventive actions.

The specific objective of the paper is to propose a novel method for flows identification and delimitation, mainly based on nondestructive measurements of water-content variations. More specifically, following this integrated method it is possible to infer the variability of volumetric water content in subbase or subgrade, to identify the probable landslide surface, to monitor the instability area and to prevent, if possible, any severe damage.

\section{Methodology}

To localise eventual instability along the road and to identify the domain of influence of the landslide, we propose here a novel methodology based on the use of GPR. The inspection for localising road sections exposed to flows is carried out at traffic speed, and it makes the methodology very efficient in comparison to any other traditional procedure. Moreover, it is very effective when it is used for the identification of the domain of influence, integrating multiple sensors and different post-processing algorithms for cross checking and evaluation.

More in depth, a three-step protocol is proposed to inspect the road pavement and structure and, finally, to support the decisions for allocating efficiently and effectively the maintenance funds.

In the first step, the instability is localised through an inspection of traffic speed using a 1-GHz GPR horn launched antenna, considering its higher productivity with respect to other ground-coupled radars. In fact, the productivity in this phase is generally about or over $300 \mathrm{Km} /$ day. Moreover, frequency is adequate in terms of resolution requirements for pavement inspection. Data are processed offline by an automatic procedure. The main output of the first step is a map of the road network where the most probable landslides are localised. These are the critical areas where the inspections are developed in the second step. Moreover, the radargrams all along the road network are available for possible additional analysis.

In the second step, GPR inspections are restricted only to the critical road sections where the probability of a landslide is higher. It makes it possible to limit the inspection at a slow speed only to some prefixed sections. The inspections are carried out using two coupled GPR dipole antennas. One antenna is $1.6 \mathrm{GHz}$ for top pavement inspection. 
The wavelength depends on the moisture content and temperature so that the resolution of this antenna is about $35-45 \mathrm{~mm}$. The signal penetrates into pavement for about $400-500 \mathrm{~mm}$. The other is a $600-\mathrm{MHz}$ antenna for sub-pavement structure diagnosis. The wavelength depends on the moisture content and temperature so that the resolution of this antenna is about $55-75 \mathrm{~mm}$. The signal penetrates about $2 \mathrm{~m}$ deep. Using these antennas, it is possible to detect both top deformations and cracks in pavement and any eventual flow under the pavement affecting the subgrade structures.

Finally, in the third step, GPR data are post-processed to identify accurately the geometry of the instability. As mentioned, the objective of post-processing is the evaluation of water-content variations in pavement or subgrade. Three different algorithms are used to post-process the GPR signal, both in the time and frequency domains. Water content is obtained both through the evaluation of the dielectric permittivity and directly from the signal scattering.

The methodology has been calibrated and validated on the road.

4. Water-content evaluation and data processing

4.1 Model for automatic inspection

The evaluation of the water-content variations in the pavement layers and in the subgrade is carried out using three different algorithms. If at least two algorithms out of three give a significant variation of water content, the road section is marked as critical and potentially exposed to a landslide.

The variation of water content between two consecutive road sections is considered critical according to the following equation:

$$
\left|\omega_{i}-\omega_{i+1}\right| \geq \Omega_{\max }
$$

where $\omega_{i}$ and $\omega_{i+1}$ are the average water content in $\%$ estimated in the consecutive road sections $i$ and $i+1$ and $\Omega_{\max }$ is the threshold of moisture variation, expressed in $\%$, that is assumed as critical. This threshold depends on the distance $D_{i}$ between the sections. Of course, as the distance $\Delta_{i}$ decreases, $\Omega_{\max }$ decreases; if the distance is relevant, a high variability in water content can be expected between the road sections.

In this study, the value of $\Omega_{\max }$ is assumed in function of the distance between two consecutive road sections $\Delta_{i}$, expressed in m, following Equation (2) [22]:

$$
\Omega_{\max }=1-e^{-\lambda \Delta_{i}}
$$

where $\lambda$ is calibrated to the value $\lambda=0.01$, thus minimising the number of false alarms. The value of $\lambda$ mainly depends on the hydraulic permittivity and porosity of soils. Following Equation (2), if the distance between two consecutive road sections is $1 \mathrm{~m}$, the moisture variation is considered automatically critical if it exceeds $1.0 \%$; if the distance is $5 \mathrm{~m}$, the critical threshold is $\Omega_{\max }=4.9 \%$ and if $\Delta_{i}$ is $10 \mathrm{~m} \Omega_{\max }$ is expected to be $9.5 \%$. For road survey purposes, the maximum distance between consecutive sections can be assumed to be usually about $15 \mathrm{~m}$. Differences of water content under the threshold are assumed to be possible also without any landslide.

The estimation of $\omega_{i}$ is done with three different algorithms. Two algorithms process the GPR signal in the time domain and are calibrated using some cores and the other algorithm operates in the frequency domain.

The algorithms in the time domain estimate the water content from the dielectric permittivity value by using the Topp equation [9]. 


\subsection{Water-content evaluation from signal amplitude attenuation}

The first algorithm is focused on the signal amplitude attenuation. The amplitude of the received signal depends on the electromagnetic characteristics of the dielectric medium. The reduction of the amplitude is a function of the dielectric permittivity. This is very sensible to the water content, being the dielectric permittivity of water $\left(\varepsilon_{\text {water }}=81\right)$, then one order of magnitude greater than the dielectric permittivity of dry soil $(\varepsilon=1)$. High-amplitude reflections indicate a high contrast between the dielectric constants of the layers.

To use the Fresnel reflection coefficient for estimating the dielectric constant of the lower layer, the dielectric constant of the top layer is needed.

In a multilayer dielectric medium comprising $\mathrm{N}$ layers, the dielectric permittivity of the generic layer i can be estimated using the following equation:

$$
\begin{array}{r}
\sqrt{\varepsilon_{1}}=k_{1} \frac{1+\frac{A_{0}}{A_{m}}}{1-\frac{A_{0}}{A_{m}}} \\
\sqrt{\varepsilon_{i}}=k_{i} \sqrt{\varepsilon_{i-1}} \frac{\frac{4 \sqrt{\varepsilon_{i-2} \varepsilon_{i-1}}}{\frac{\varepsilon_{i-2}-\varepsilon_{i-1}}{4 \sqrt{\varepsilon_{i-2} \varepsilon_{i-1}}}}-\frac{A_{i-1}}{A_{i-2}}}{A_{i-2}-\varepsilon_{i-1}}+\frac{A_{i-1}}{A_{i-2}}
\end{array}
$$

where $\mathrm{i}$ is the sequence number of layers, $\mathrm{N}$ is the total number of layers, $\mathrm{A}_{\mathrm{m}}$ is the reflection amplitude of the metal plate and $A_{i}$ is the reflection amplitude from the top of layer $i$. The coefficient $k_{i}$ is a calibration factor that takes into account the characteristics of signal propagation in the specific medium [20]. The equations can be used unless knowing the values of the thickness of the layers and the velocity of the electromagnetic wave through the dielectric medium.

\subsection{Water-content evaluation from signal-propagation velocity}

The average value of the dielectric permittivity can be computed according to the following equation:

$$
\varepsilon=\left(\frac{c \cdot \Delta t}{h}\right)^{2}(5)
$$

where $\mathrm{c}$ is the signal velocity in void, Dt is the time delay of reflection arrival and $\mathrm{h}$ is the thickness of the layer, being the signal propagation velocity in the medium $v=c /(\varepsilon)^{0.5}$.

Once the dielectric permittivity is estimated using Equation (4) or Equation (5), the soil water content is computed using the Topp equation [9], which is the following empirical relationship relating dielectric permittivity to water content:

$$
\omega=-0.053+0.0292 \varepsilon-5.5 \cdot 10^{-4} \varepsilon^{2}+4.3 \cdot 10^{-6} \varepsilon^{3}
$$

\subsection{Water-content evaluation from signal scattering}

The third algorithm used to estimate water content is focused on the scattering of the signal on the basis of the Rayleigh theory [21]. The main advantage of this method is that it does not require any field calibration. The signal is processed in the frequency domain; this method is based on the Rayleigh scattering according to the Fresnel theory. The scattering produces a nonlinear frequency modulation of the electromagnetic signal, where the modulation is a function of the water content [23]. The following regression law is proposed to predict moisture content (v), expressed in $\%$, from the value of the peak of 
frequency $\left(\mathrm{f}_{\mathrm{P}}\right)$, expressed in $\mathrm{Hz}$ :

$$
\omega=\left(A-f_{P}\right) / B
$$

where A and B are the regression coefficients calibrated with experimental data obtained from laboratory tests and carried out on different type of soils. Those are univocally characterised by a couple of A and B values case by case. The value of the peak of frequency is extracted from the corresponding frequency spectrum of the reflected radar signal the fast Fourier transform.

The values of A vary from $5.3 \times 10^{8}$ to $7.0 \times 10^{8}$ and the values of $\mathrm{B}$ vary from $1.1 \times 10^{7}$ to $2.3 \times 10^{7}$, depending on the investigated type of soil [21]. This algorithm gives directly the water content through Equation (7).

\section{Case study}

The proposed methodology has been tested on a rural road network located in a district $100 \mathrm{~km}$ north from Rome. The network is in a hilly and mountainous environment and it is frequently exposed to landslides, especially flows. In the first step of the assessment process, about $200 \mathrm{~km}$ per day of the network has been inspected using the 1-GHz horn antenna at traffic speed (Figure 1).

From the post-processing of data, seven most critical areas that are probably affected by flows have been identified. The water content variability and the horizontality of layers are checked. The visual inspection and the existing literature confirmed the presence of landslide, typically flows of clayey or silty soil masses over a limestone bedrock.

Here the outcome of one of those seven cases is presented, which shows the effectiveness of the protocol.

The road section is about $2000 \mathrm{~m}$ long. The road comprises two 3.5-m-wide lanes, the shoulders are $0.50 \mathrm{~m}$, the total paved area is $8.0 \mathrm{~m}$ wide.

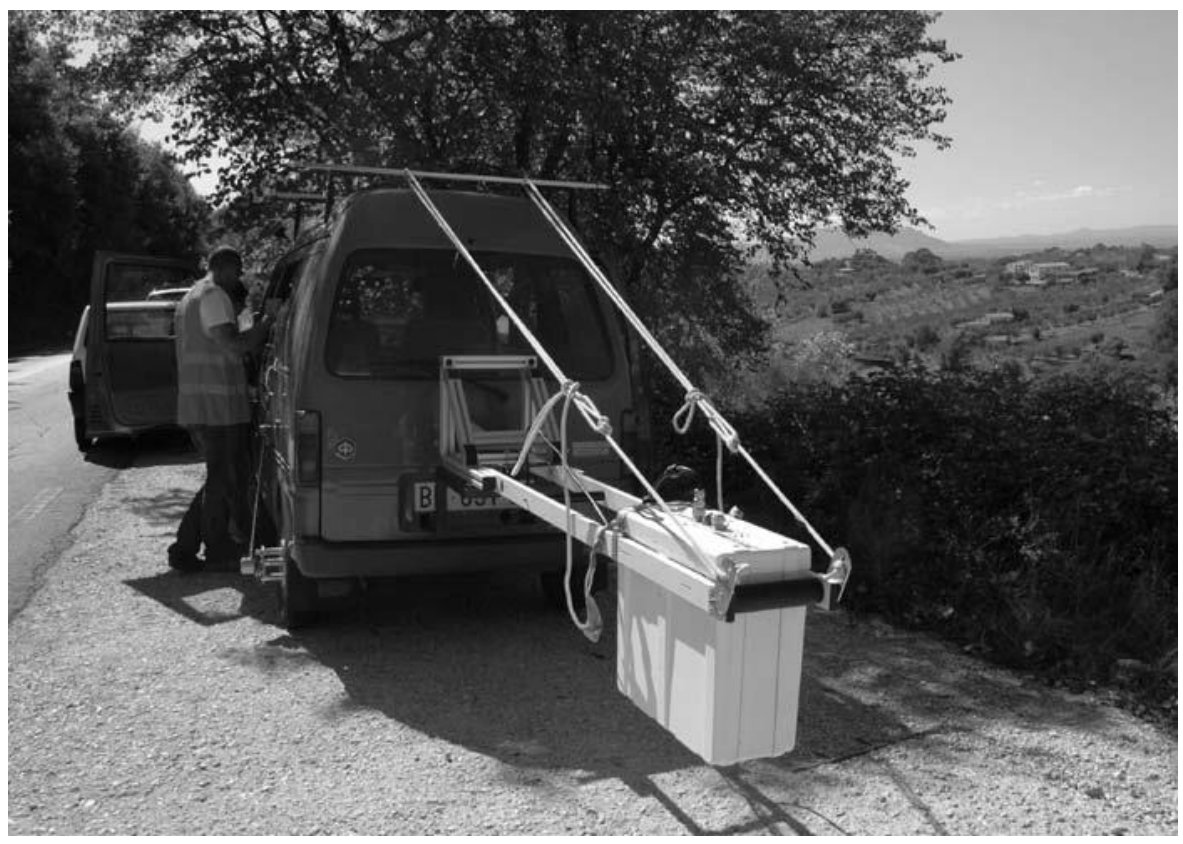

Figure 1. GPR horn antenna. 

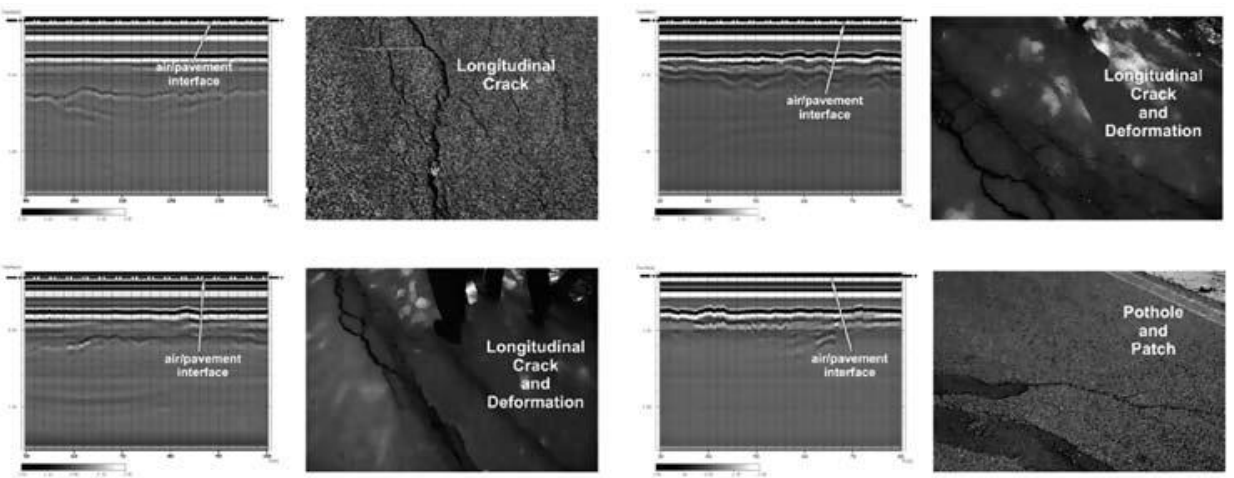

Figure 2. Localised damages and radargrams $(1 \mathrm{GHz})$.

A wide landslide in a resting stage affects the road causing degradation of soils and damage to the pavement.

The serviceability of pavement is low because of several diffused cracks and longitudinal deformations.

In some places the cracks are so relevant that the paved surface has a sort of step (Figure 2). In Figure 2, some radargrams acquired using the $1-\mathrm{GHz}$ antenna are shown, where it is evident that the pavement layers appear to be not horizontal.

In the second step, the 2000-m road stretch has been inspected using the two dipole antennas $(600 \mathrm{MHz}$ and $1.6 \mathrm{GHz})$. The GPR is dragged walking at a smart pace (speed $3-4 \mathrm{~km} / \mathrm{h}$ ) along the right and left lane.

Transversal GPR scans have been executed very $50 \mathrm{~m}$ along the road. The longitudinal scans are useful to detect any variation of moisture along a lane; comparing the left lane
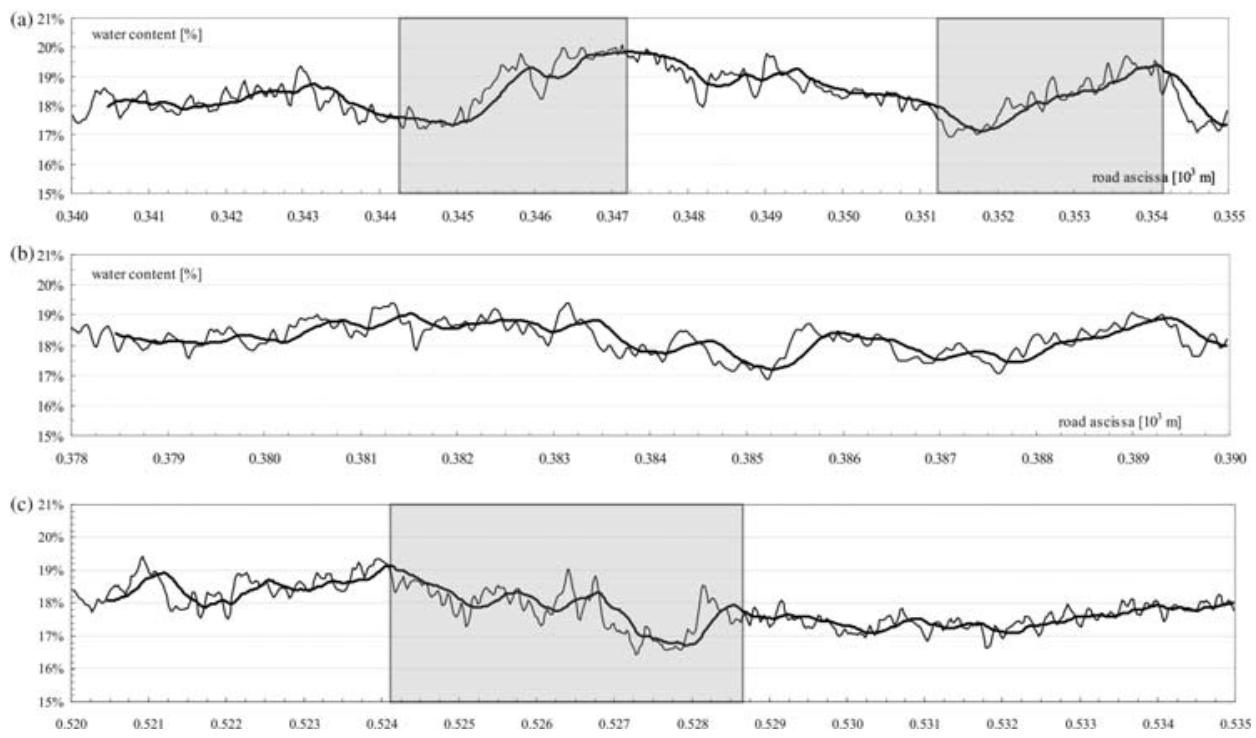

Figure 3. Water content profiles along the road: (a) 340-355 $\mathrm{m}$ in grey, the critical variations of moisture; (b) $378-390 \mathrm{~m}$, no critical variations of moisture; (c) $520-535 \mathrm{~m}$ in grey, the critical variations of moisture. 

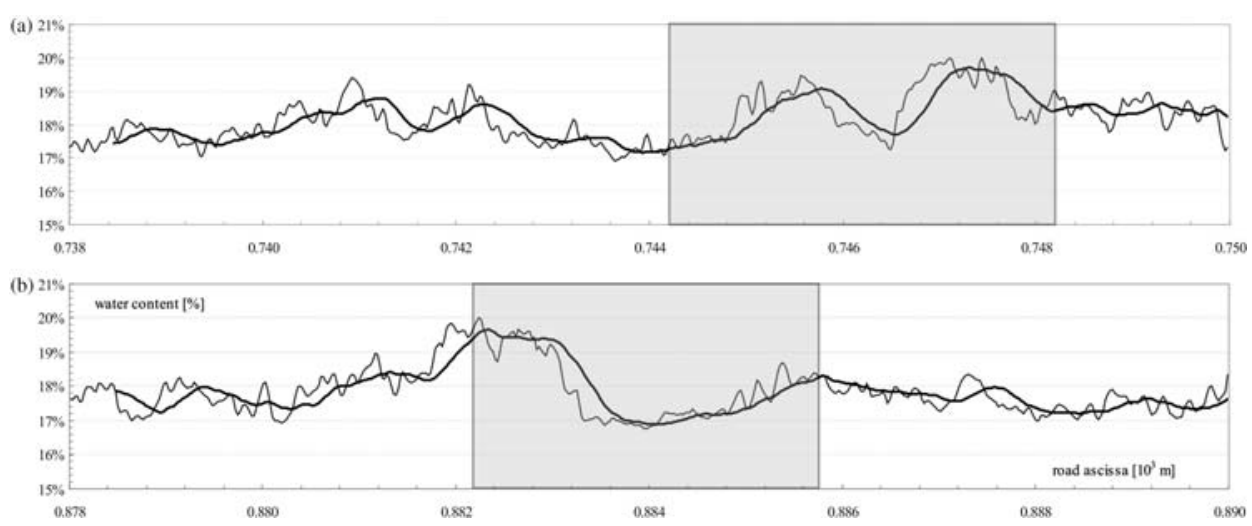

Figure 4. Water content profiles of right and left lane: (a) $738-750 \mathrm{~m}$ in grey, the zones where moisture is significantly different between left and right lane; (b) $878-890 \mathrm{~m}$ in grey, the zones where moisture is significantly different between left and right lane.

scan and the right lane scan, it is possible to detect moisture variation across the carriageway. The transversal scans have been used for validation and cross checking.

GPR signals have been finally post-processed (third step) to evaluate the variation of water content all along the $2000 \mathrm{~m}$ of the road stretch. For instance, Figure 3(a)-(c) shows some moisture profiles along the road. They are discussed in the next chapter.

Figure 4(a),(b) compares the water-content profiles of right and left lanes in the case in which the moisture is different.

\section{Discussion}

The proposed method for road inspection results is much more efficient than any other traditional one. First of all, the traditional destructive technologies (DTs) are used in restricted areas or work zones and they cannot give a continuous inspection of the road network, as it is expected from the first step of the proposed protocol. In addition, their productivity can be estimated, at an average of $10^{2} \mathrm{~m}$ of road per day, while $10^{3} \mathrm{~m}$ of road per day are reasonably inspected using GPR in the second step of the protocol. Then, the productivity can be estimated one order of magnitude greater than using DTs.

The traditional inspection needs some traffic restrictions for coring and measuring, otherwise in the second step of the protocol very low traffic interferences are expected. It makes the proposed protocol much safer.

In addition, the DTs are used for inspection of subbase and subgrade as a damage is visible on the pavement. Conversely in the first step, the proposed protocol allows to extend the inspection along the entire road network for identifying the most probable cases where some landslide is occurring. It is also possible to analyse the horizontality of interfaces and the water-content distribution in the deeper layers in the case where there is no visible effect on the top of the pavement.

According to Equations (1) and (2), Figure 3(a) - (c) shows the anomalous variations of water content that can be automatically detected. As the difference between the water contents does not exceed the value of $\Omega_{\max }$, the procedure does not mark any critical section as shown in Figure 3(b). However, in this last case the value of moisture results relatively high and is compatible with the presence of a wide flow all along the stretch. In fact, this is the case as it is shown in Figure 5. 


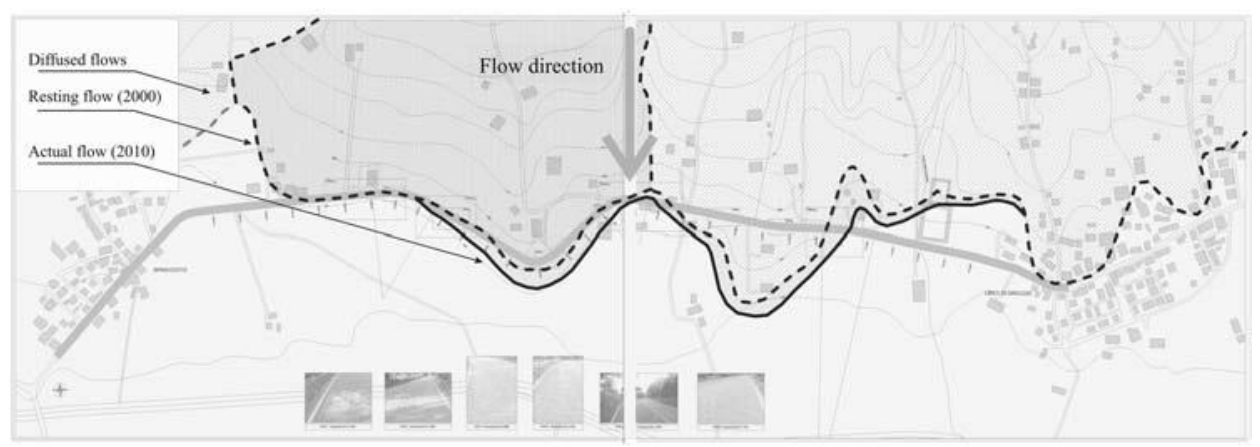

Figure 5. Landslide geometry, direction of flow and interferences with the road.

In addition, the water-content profiles along the right and left lanes of the road are put in evidence when there are some discrepancies, as shown in Figure 4(a),(b). It happens if the water content varies transversally under the pavement. It is the case in which the front of the landslide intersects the road, generating a different water content between the sub-asphalt layers in the right and left lanes.

In this specific case study, the landslide was classified in 2000 by the Regional Agency in a resting stage and the geometry of the mass of soil was defined as in Figure 5.

Following the results of the GPR inspection that allow to identify where the water content rapidly changes and where the front of the landslide is expected to be, it is possible to redraw and update the current geometry of the landslide. This is shown in Figure 5.

It is evident that a slow flow of the soil mass has occurred in these approximately 10 years. This is the prevalent cause of the pavement damage.

The landslide front intersects the road three times (Figure 5). Referring to the road abscissa, it can be noted that the front of the landslide is localised approximately in the sections between 250 and $370 \mathrm{~m}$ (e.g. Figure 3(a)), 740 and $950 \mathrm{~m}$ (Figure 4(a),(b)) and finally 1200 and $1350 \mathrm{~m}$. The stretch of road approximately from 370 to $1200 \mathrm{~m}$ is mainly interested by the landslide. According to this, in this area the value of moisture is generally over $18-19 \%$ (Figure 3(b),(c)).

\section{Conclusions}

In conclusion, the proposed procedure results, on the basis of GPR inspection, are much more efficient in respect to any traditional method or procedure. The productivity is so promising that it is expected that hundreds of kilometres of rural road networks can be inspected in a week. The case study demonstrates that it is possible to identify where the water content rapidly changes and where the front of the landslide is expected to be. This result makes it possible to support the decision-making process and to address the maintenance funds in the most sustainable and efficient way. In addition, the accuracy of the radar inspection is also strategic for identifying and planning the most effective rehabilitation and maintenance works. Finally, the methodology is very useful also in preventing the severe damages that are not yet visible on the top surface of the pavement.

\section{References}

[1] D.J. Varnes, Slope movement types and processes, in Landslides, Analysis and Control. Transportation Research Board Special Report No. 176, R.L. Schuster and R.J. Krizek, eds., National Academy of Sciences, Washington, D.C., 1978, pp. 11-33. 
[2] D.M. Cruden and D.J. Varnes, Landslides types and processes, in Landslides: Investigation and Mitigation. Transportation Research Board Special Report No. 247, A.K. Turner and R.L. Schuster, eds., National Academy Press, Washington, D.C., 1996, pp. 36-75.

[3] J. Hruska and F. Hubatka, Landslide investigation and monitoring by a high-performance ground penetrating radar system, Proceedings of the 8th International Conference on Ground Penetrating Radar, Vol. 4084, Gold Coast, Australia, SPIE, Bellingham, WA, USA, 2000.

[4] W.A. Bamhardt and R.E. Kayen, Radar structure of earthquake-induced, coastal landslides in Anchorage, Alaska, Environ. Geosci. 7 (2000), pp. 38-45.

[5] O.N. Cutlac and J.M. Maillol, Study of Holocene landslide deposits by comparison of GPR, refraction seismic and electrical resistivity data, Proceedings of the 10th International Conference on Ground Penetrating Radar, Delft, The Netherlands, New York, IEEE Press, 2004.

[6] K.H. Roch, W. Chwatal, and E. Brückl, Potential of monitoring rock fall hazards by GPR: Considering as example the results of Salzburg, Landslides 3 (2006), pp. 87-94.

[7] C. Ékes and P. Friele, Ground penetrating radar and its use in forest road stability analysis, Proceedings of the 10th International Conference on Ground Penetrating Radar, Delft, The Netherlands, New York, IEEE Press, 2004.

[8] A. Benedetto and S. Pensa, Indirect diagnosis of pavement structural damages using surface GPR reflection techniques, J. Appl. Geophys. 62 (2007), pp. 107-123.

[9] G.C. Topp, J.L. Davis, and A.P. Annan, Electromagnetic determination of soil water content: measurements in coaxial transmission lines, Water Resour. Res. 16 (1980), pp. 574-582.

[10] S.P. Friedman, A saturation degree-dependent composite spheres model for describing the effective dielectric constant of unsaturated porous media, Water Resour. Res. 34 (1998), pp. 2949-2961.

[11] K. Grote, S. Hubbard, and Y. Rubin, GPR monitoring of volumetric water content in soils applied to highway construction and maintenance, The Leading Edge 21 (2002), pp. 482-485.

[12] K. Grote, S. Hubbard, and Y. Rubin, Field-scale estimation of volumetric water content using GPR groundwave techniques, Water Resour. Res. 39 (2003), pp. 1321-1335.

[13] S. Hubbard, K. Grote, and Y. Rubin, Estimation of nearsubsurface water content using high frequency GPR ground wave, The Leading Edge 21 (2002), pp. 552-559.

[14] J.A. Huisman, S.S. Hubbard, J.D. Redman, and A.P. Annan, Measuring soil water content with ground penetrating radar: A review, Vadose Zone J. 2 (2003), pp. 476-491.

[15] D.A. Robinson, S.B. Jones, J.M. Wraith, D. Or, and S.P. Friedman, A review of advances in dielectric and electrical conductivity measurement in soils using time domain reflectometry, Vadose Zone J. 2 (2003), pp. 444-475.

[16] G. Serbin and D. Or, Near-surface soil water content measurements using horn antenna radar: methodology and overview, Vadose Zone J. 2 (2003), pp. 500-510.

[17] A. Fiori, A. Benedetto, and M. Romanelli, Application of the effective medium approximation for determining water contents through GPR in coarse-grained soil materials, Geophys. Res. Lett. 32 (2005), doi:10.1029/2005GL022555.

[18] A. Benedetto and F. Benedetto, GPR experimental evaluation of subgrade soil characteristics for rehabilitation of roads, Proceedings of the 9th International Conference on Ground Penetrating Radar, Santa Barbara, CA, SPIE, Bellingham, WA, USA, 2002.

[19] A. Benedetto, Theoretical approach to electromagnetic monitoring of road pavement, Proceedings of the 10th International Conference on Ground Penetrating Radar, Delft, The Netherlands, New York, IEEE Press, 2004.

[20] C. Huang and Y. Su, A new GPR calibration method for high accuracy thickness and permittivity measurement of multi-layered pavement, Proceedings of the 10th International Conference on Ground Penetrating Radar, Delft, The Netherlands, New York, IEEE Press, 2004.

[21] A. Benedetto, Water content evaluation in unsaturated soil using GPR signal analysis in the frequency domain, J. Appl. Geophys. 71 (2010), pp. 26-35.

[22] A. Benedetto, G. Schettini, F. Tosti, and C. Twizere, Evaluation of geotechnical stabilityof road using GPR, Proceedings of the 6th International Workshop on Advanced Ground Penetrating Radar (IWAGPR), Aachen, Germany, New York, IEEE Press, 2011.

[23] S. Chakraborti, Verification of the Rayleigh scattering cross section, Am. J. Phys. 75 (2007), pp. $824-826$. 